\title{
SPLITTING TWISTED SUMS OF NUCLEAR KÖTHE SPACES
}

\section{KAISA NYBERG}

1. Introduction. In the present paper we continue the work started in [2]. There we showed that the middle space $X$ in a short exact sequence $0 \rightarrow E \rightarrow X \rightarrow F \rightarrow 0$ of nuclear Fréchet spaces can be represented as the direct sum of $E$ and $F$ equipped with the topology "twisted" by a sequence of linear maps from a dense subspace of $F$ to $E[2 \text {, Theorem 2.5] }]^{1)}$. This representation was used to give a sufficient condition for splitting short exact sequences of nuclear Köthe spaces. In this paper we shall prove the necessity of this condition.

Other splitting conditions have been introduced by D. Vogt in [6]. One of these, called $\left(\mathrm{S}^{*}\right)$, which is known to be necessary, was recently proved by J. Krone and Vogt to be sufficient for nuclear Köthe spaces [3]. We shall directly show that $\left(S^{*}\right)$ implies our splitting condition; thus we have a second proof for the sufficiency of $\left(\mathrm{S}^{*}\right)$ essentially contained in [2].

Applications of the sufficient splitting condition can be found in [2] and [4] (see also [1]), and in [3]. We refer to [2] for the unexplained terminology.

2. The splitting. In nuclear Fréchet spaces $E$ and $F$ we consider fundamental systems of continuous seminorms $\|\cdot\|_{p}$ such that the corresponding unit balls form neighbourhood bases of the origin. In the dual space $E^{\prime}$ we have $R \cup\{\infty\}$-valued norms defined as follows:

$$
\left\|x^{\prime}\right\|_{p}=\sup \left\{\left|\left\langle x, x^{\prime}\right\rangle\right|\|x\|_{p} \leqq 1, x \in E\right\} .
$$

Assume that $E$ has a basis $\left(e_{n}\right)$ and let $\left(e_{n}^{\prime}\right)$ be the corresponding coordinate functionals. By virtue of nuclearity, the topology of $E$ is determined by the fundamental system of seminorms

$$
\left.\|x\|_{p}=\sup _{n \in N}\left|\left\langle x, e_{n}^{\prime}\right\rangle\right|\left\|e_{n}\right\|_{p} \quad \text { (or }\|x\|_{p}=\sum_{n=1}^{\infty}\left|\left\langle x, e_{n}^{\prime}\right\rangle\right|\left\|e_{n}\right\|_{p}\right) .
$$

We call these the sup-seminorms (or sum-seminorms) in $E$ with respect to $\left(e_{n}\right)$.

1) The proof of this theorem contains a mistake which was pointed out to the authors by Pawel Domański. To correct the theorem it is sufficient (and also necessary) to assume that the second space $F$ has a continuous norm. For example, the short exact sequence $0 \rightarrow s \rightarrow s \rightarrow s^{N} \rightarrow 0$ constructed in [5, Lemma 1.6] cannot be represented in this way. 
Let us recall the following splitting condition given in [2]:

$$
\begin{gathered}
\forall \sigma: N \rightarrow N \exists \varrho: N \rightarrow N \forall p, q, p>q, x^{\prime} \in E^{\prime}, y \in F \\
\sum_{r=q}^{p-1}\left\|x^{\prime}\right\|_{r}\|y\|_{\sigma(r)} \leqq\left\|x^{\prime}\right\|_{q}\|y\|_{\varrho(q)}+\left\|x^{\prime}\right\|_{p}\|y\|_{\varrho(p)} .
\end{gathered}
$$

An equivalent formulation of this condition is

$$
\begin{gathered}
\forall \sigma: N \rightarrow N \exists \varrho: N \rightarrow N \forall p, q, p>q, x^{\prime} \in E^{\prime}, y \in F \\
\max _{q \leqq r<p}\left\|x^{\prime}\right\|_{r}\|y\|_{\sigma(r)} \leqq\left\|x^{\prime}\right\|_{q}\|y\|_{\varrho(q)}+\left\|x^{\prime}\right\|_{p}\|y\|_{\varrho(p)} .
\end{gathered}
$$

To verify that (TS') implies (TS), let $\sigma: N \rightarrow N$ be arbitrary. Let $\varphi$ be such that $\|y\|_{\sigma(r)} \leqq 2^{-r}\|y\|_{\varphi(r)}$ for all $r \in N$ and $y \in F$. Now use (TS') for $\varphi$ to get $\varrho$. Then this $\varrho$ satisfies (TS) with $\sigma$.

Lemma 2.1. If the nuclear Fréchet spaces $E$ and $F$ have bases, then (TS) implies that $F$ admits a continuous norm.

Proof. Let $\left(e_{n}\right)$ and $\left(f_{m}\right)$ be bases in $E$ and $F$. Let $\sigma$ and $\varrho$ be such that (TS) is satisfied, and assume that there is no continuous norm in $F$. Then there is $q$ and $m$ such that $\left\|f_{m}\right\|_{e(q)}=0$. By (TS) we have

$$
\left\|f_{m}\right\|_{\sigma(r)}\left\|e_{n}^{\prime}\right\|_{r} \leqq\left\|f_{m}\right\|_{Q(p)}\left\|e_{n}^{\prime}\right\|_{p}
$$

for all $n, p$, and $r, p>r \geqq q$. There is $r_{0}$ such that $\left\|f_{m}\right\|_{\sigma(r)}>0$ for every $r \geqq r_{0}$. Also, for every $n$, there is $p$ such that $\left\|e_{n}^{\prime}\right\|_{p}<\infty$. Thus it follows from (1) that $\left\|e_{n}^{\prime}\right\|_{r}<\infty$ for every $n$ and $r, r \geqq r_{0}$. By nuclearity there is $p, p>r \geqq r_{0}$, such that

$$
\lim _{n \rightarrow \infty}\left\|e_{n}^{\prime}\right\|_{p} /\left\|e_{n}^{\prime}\right\|_{r}=\lim _{n \rightarrow \infty}\left\|e_{n}\right\|_{r} /\left\|e_{n}\right\|_{p}=0 .
$$

Consequently, by (1), $\left\|f_{m}\right\|_{\sigma(r)}=0$ for all $r \geqq r_{0}$, which cannot be true since $F$ is Hausdorff.

We have the following theorem.

Theorem 2.2. Assume that the nuclear Fréchet spaces $E$ and $F$ have bases $\left(e_{n}\right)$ and $\left(f_{m}\right)$. Then the following three assertions are equiralent:

(i) (TS) holds for every fundamental system of sum-seminorms $\|\cdot\|_{p}$ in $E$ such that $\lim _{n \rightarrow \infty}\left\|e_{n}\right\|_{p} /\left\|e_{n}\right\|_{p+1}=0, p \in N$.

(ii) There is a fundamental system of seminorms in E such that (TS) is satisfied.

(iii) $F$ admits a continuous norm and every short exact sequence $0 \rightarrow E \rightarrow X \rightarrow F \rightarrow 0$ splits. 
Proof. The implication (ii) $\Rightarrow$ (iii) is Theorem 4.1. of [2] together with the previous lemma. It remains to prove that (iii) implies (i).

We shall first construct a suitable short exact sequence. To do this, we consider the sup-seminorms in $E$. Let $c_{r}>0$ be such that $\sum_{r=1}^{\infty} c_{r} \leqq 1$. Now fix $r$ and $m$ for a moment and let $q<r$. Since $F$ has a continuous norm and $E$ is nuclear, there is an index $n(m, r, q)$ such that

$$
\left.\left\|e_{n}^{\prime}\right\|_{r} /\left\|e_{n}^{\prime}\right\|_{q} \leqq c_{r}\left\|f_{m}\right\|_{\sigma(q)}\right)\left\|f_{m}\right\|_{\sigma(r)}
$$

for $n>n(m, r, q)$. Thus there exist indices $n(m, r)$ such that $n(m, r)<n(m, r+1)$ and $n(m, r) \geqq \max _{1 \leqq q<r} n(m, r, q)$.

We denote the algebraic linear span of the basis $\left(f_{m}\right)$ by $F_{0}$ and define linear maps $A_{r}: F_{0} \rightarrow E$ as follows:

$$
\begin{gathered}
A_{1} f_{m}=0, \quad m \in N, \\
A_{r+1} f_{m}=A_{r} f_{m}+\left\|f_{m}\right\|_{\sigma(r)} \sum_{n=1}^{n(m, r)}\left\|e_{n}^{\prime}\right\|_{r} e_{n} .
\end{gathered}
$$

Since $\left\|A_{r+1} f_{m}-A_{r} f_{m}\right\|_{r}=\left\|f_{m}\right\|_{\sigma(r)}$, the maps $A_{r}, r \in N$, define a short exact sequence

$$
0 \rightarrow E \rightarrow E \hat{x}_{\left(A_{r}\right)} F_{0} \rightarrow F \rightarrow 0,
$$

where the middle space is the completion of the direct sum $E \times F$ equipped with the topology defined by the seminorms

$$
(x, y) \mapsto\|(x, y)\|_{r}=\left\|x-A_{r} y\right\|_{r}+\|y\|_{\sigma(r)}
$$

[2, Lemma 2.3 and Theorem 2.4]. By the assumption (3) splits; consequently, by [2, Theorem 2.6], there exists a map $A: F_{0} \rightarrow E$ and a function $\varrho: N \rightarrow N$ such that for every $r$ and $m$

$$
\left\|A f_{m}-A_{r} f_{m}\right\|_{r} \leqq\left\|f_{m}\right\|_{Q}(r) .
$$

Now let $q, p, q<p$ and $m$ be arbitrarily fixed. We have three possibilities.

(i) $n \leqq n(m, q)$. By the definition of the maps $A_{r}$ we have

$$
\begin{gathered}
\sum_{r=q}^{p-1}\left\|f_{m}\right\|_{\sigma(r)}\left\|e_{n}^{\prime}\right\|_{r}=\sum_{r=q}^{p-1}\left\langle A_{r+1} f_{m}-A_{r} f_{m}, e_{n}^{\prime}\right\rangle=\left\langle A_{p} f_{m}, e_{n}^{\prime}\right\rangle-\left\langle A_{q} f_{m}, e_{n}^{\prime}\right\rangle \\
=\left\langle A_{p} f_{m}-A f_{m}, e_{n}^{\prime}\right\rangle+\left\langle A f_{m}-A_{q} f_{m}, e_{n}^{\prime}\right\rangle \leqq\left\|f_{m}\right\|_{\ell(p)}\left\|e_{n}^{\prime}\right\|_{p}+\left\|f_{m}\right\|_{\varrho(q)}\left\|e_{n}^{\prime}\right\|_{q},
\end{gathered}
$$

where the last inequality is obtained by (4).

(ii) $n(m, p)<n$. In this case we use (2):

$$
\sum_{r=q}^{p-1}\left\|f_{m}\right\|_{\sigma(r)}\left\|e_{n}^{\prime}\right\|_{r} \leqq \sum_{r=q}^{p-1} c_{r}\left\|f_{m}\right\|_{\sigma(q)}\left\|e_{n}^{\prime}\right\|_{q} \leqq 2\left\|f_{m}\right\|_{\sigma(q)}\left\|e_{n}^{\prime}\right\|_{q} .
$$

Now choose $\varrho$ such that $2\|\cdot\|_{\sigma(q)} \leqq\|\cdot\|_{\varrho(q)}$, and we have (TS).

(iii) $n(m, q)<n \leqq n(m, p)$. This case is a combination of (i) and (ii). There is an $r_{0}, q \leqq r_{0}<p$ such that $n\left(m, r_{0}\right)<n \leqq n\left(m, r_{0}+1\right)$. Let us devide the sum

$$
\sum_{r=q}^{p-1}\left\|f_{m}\right\|_{\sigma(r)}\left\|e_{n}^{\prime}\right\|_{r}=\sum_{r=q}^{r_{0}}+\sum_{r=r_{0}+1}^{p-1}
$$


into two parts and apply (ii) to the first part. As to the second part, we observe that, by the definition of the maps $A_{r}$, we have $\left\langle A_{r+1} f_{m}-A_{r} f_{m}, e_{n}^{\prime}\right\rangle=0$ for $q \leqq r \leqq r_{0}$ because $n>n\left(m, r_{0}\right) \geqq n(m, r)$. Thus

$\sum_{r=r_{0}+1}^{p-1}\left\|f_{m}\right\|_{\sigma(r)}\left\|e_{n}^{\prime}\right\|_{r}=\sum_{r=r_{0}+1}^{p-1}\left\langle A_{r+1} f_{m}-A_{r} f_{m}, e_{n}^{\prime}\right\rangle=\sum_{r=q}^{p-1}\left\langle A_{r+1} f_{m}-A_{r} f_{m}, e_{n}^{\prime}\right\rangle$

and we can continue as in (i). Finally, increasing $\varrho$ we put the parts together to obtain (IS) for the basis vectors.

Let us now assume that we have the sum-seminorms in $E$. Then

$$
\left\|x^{\prime}\right\|_{p}=\sup _{n \in N}\left|\left\langle e_{n}, x^{\prime}\right\rangle\right|\left\|e_{n}^{\prime}\right\|_{p} .
$$

Since (TS) does not depend on the chosen system of seminorms in $F$, we may consider the sup-norms. Now it is easy to see that (TS) holds for all vectors $x^{\prime}$ in $E^{\prime}$ and $y$ in $F$.

It might be of some interest to compare (IS) with the following condition:

$$
\forall u \exists n_{0}, k \forall K, m \quad \exists n:\left\|x^{\prime}\right\|_{k}\|y\|_{m} \leqq\left\|x^{\prime}\right\|_{u}\|y\|_{n_{0}}+\left\|x^{\prime}\right\|_{K}\|y\|_{n}, \quad x^{\prime} \in E^{\prime}, y \in F,
$$

which, as a result of completely different methods, has also been proved to characterize the splitting of every short exact sequence $0 \rightarrow E \rightarrow X \rightarrow F \rightarrow 0$ at least for nuclear Köthe spaces $E$ and $F[3]$.

It is easy to show directly that $\left(\mathrm{S}^{*}\right)$ implies (TS).

Proposition 2.3. If $\left(\mathrm{S}^{*}\right)$ holds for given fundamental systems of seminorms in $E$ and $F$, then, passing to a subsequence of the system of seminorms in $E$, we have ( $\left.\mathrm{TS}^{\prime}\right)$.

Proof. We use $\left(\mathrm{S}^{*}\right)$ and choose for every $u$ indices $n_{0}$ and $k=k(u)$ such that

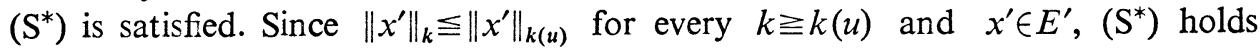
for every $k \geqq k(u)$. Thus, passing to a sequence of seminorms in $E$, we have

$$
\forall u \quad \exists n_{0} \quad \forall k \geqq u+1 \quad \forall K, m \quad \exists n:\left\|x^{\prime}\right\|_{k}\|y\|_{m} \leqq x^{\prime}\left\|_{u}\right\| y\left\|_{n_{0}}+\right\| x^{\prime}\left\|_{K}\right\| y \|_{n} .
$$

Now let $\sigma: N \rightarrow N$ be a function and $q<r<p$. We use (5) to get $n_{0}=\varphi(q)$ such that for every $k=r, m=\sigma(r)$ and $K=p$ there is $n=n(r, p, \sigma)$ such that

$$
\left\|x^{\prime}\right\|_{r}\|y\|_{\sigma(r)} \leqq\left\|x^{\prime}\right\|_{q}\|y\|_{\varphi(q)}+\left\|x^{\prime}\right\|_{p}\|y\|_{n} .
$$

We set $\varrho(p)=\max _{r<p} n(r, p, \sigma)$; then for every $r, q<r<p$, we have

$$
\left\|x^{\prime}\right\|_{r}\|y\|_{\sigma(r)} \leqq\left\|x^{\prime}\right\|_{q}\|y\|_{\varphi(q)}+\left\|x^{\prime}\right\|_{p}\|y\|_{\ell(p)} .
$$

Now we increase $\varrho$ if necessary to obtain (TS'). 


\title{
References
}

[1] Hebbecker, J.: Auswertung der Splittingbedingungen $S_{1}$ und $S_{2}$ für Potenzreihenräume und $L_{f}$-räume. - Diplomarbeit, Wuppertal, 1984.

[2] Ketonen, T., and K. Nyberg: Twisted sums of nuclear Fréchet spaces. - Ann. Acad. Sci. Fenn. Ser. A I Math. 7, 1982, 323-335.

[3] Krone, J., and D. VoGt: The splitting relation for Köthe spaces. - Math. Z. 190, 1985, 387-400.

[4] Nyberg, K.: Tameness of pairs of nuclear power series spaces and related topics. - Trans. Amer. Math. Soc. 283:2, 1984, 645-659.

[5] Vogr, D.: Characterisierung der Unterräume von s. - Math. Z. 155, 1977, 109-177.

[6] VoGT, D.: On the functors $\operatorname{Ext}^{1}(E, F)$ for Fréchet spaces. - Preprint.

\author{
University of Helsinki \\ Department of Mathematics \\ SF-00100 Helsinki \\ Finland
}

Received 7 March 1985 